\title{
Transcatheter Aortic Valve Dislocation in Left Ventricular Outflow Tract with Successful Repositioning Using “Double Snare” Technique
}

\author{
Matjaž Bunc ${ }^{1 *}$, Luka Vitez¹, Simon Terseglav¹, Tadej Žlahtič¹, Gian Paolo Ussia² \\ ${ }^{1}$ Clinical Department for Cardiology, University Medical Centre Ljubljana, Ljubljana, Slovenia \\ ${ }^{2}$ Department of Medicine, Interventional Cardiology Unit, University Campus Bio-Medico of Rome, Rome, Italy \\ Email: *mbuncek@yahoo.com
}

How to cite this paper: Bunc, M., Vitez, L., Terseglav, S., Žlahtič, T. and Ussia, G.P. (2021) Transcatheter Aortic Valve Dislocation in Left Ventricular Outflow Tract with Successful Repositioning Using "Double Snare" Technique. International Journal of Clinical Medicine, 12, 7-13.

https://doi.org/10.4236/ijcm.2021.121002

Received: November 11, 2020

Accepted: January 11, 2021

Published: January 14, 2021

Copyright $\odot 2021$ by author(s) and Scientific Research Publishing Inc. This work is licensed under the Creative Commons Attribution International License (CC BY 4.0).

http://creativecommons.org/licenses/by/4.0/

\begin{abstract}
Background: Transcatheter aortic valve implantation (TAVI) is a widely used treatment of severe aortic stenosis. Implantation of a self-expanding valve into a dense calcified aortic annulus can be challenging and may result in device malposition and malfunction. Aim: The aim of our case report is to present a novel technique of transcatheter aortic valve dislocation treatment. Case presentation: An 86-year-old woman with severely calcified aortic valve underwent TAVI using a 27-mm self-expanding Portico valve (Abbott Vasc, USA). In the last phase of implantation, the valve dislocated deep into the left ventricular outflow tract resulting in significant paravalvular regurgitation and patient instability. Repositioning of the valve with a single snare was ineffective because of severe aortic ring calcifications. A novel "double snare" technique was applied and the valve was successfully repositioned upward with an excellent anatomic and haemodynamic result. Conclusion: "Double snare" technique can be an effective strategy for repositioning of deeply implanted self-expanding transcatheter aortic valves. It represents an efficient bailout strategy in case of single snare approach failure, especially in cases of severe aortic ring calcifications.
\end{abstract}

\section{Keywords}

Aortic Valve, Transcatheter Aortic Valve Replacement, Complications, Aortic Valve Stenosis

\section{Introduction}

Transcatheter aortic valve implantation (TAVI) is a widely used treatment of aortic stenosis. Prosthesis dislocation immediately after TAVI deployment is a 
rare complication and is usually a result of prosthesis-annulus mismatch associated with too high or too low implantation, ejection of the device by an effective ventricular contraction during deployment or lack of significant calcifications for prosthesis anchoring [1] [2] [3]. Deployment of self-expanding valves requires a slow release of tension that builds up in the delivery system. Caution is thus warranted since any undesirable motion from the patient or operator can lead to a dislocation [4]. Most frequently, the valve embolizes upward into the ascending aorta. In such case, upward snaring and repositioning of the embolized valve and placement of a second valve is the usual strategy [2]. In case of downward intra-ventricular valve dislocation, the situation is much more challenging with the final valve position being of outmost importance. Here, we present a case of transcatheter aortic valve dislocation in the left ventricular outflow tract (LVOT) with successful repositioning using a novel percutaneous approach.

\section{Case Presentation}

An 86-year old woman with arterial hypertension and osteoporosis was admitted for treatment of severe symptomatic aortic stenosis. Transthoracic echocardiography (TTE) showed normal left ventricular systolic function, impaired left ventricular diastolic function and a calcified trileaflet aortic valve with severe aortic stenosis (aortic valve area $0.7 \mathrm{~cm}^{2}$, maximal velocity of $3.9 \mathrm{~m} / \mathrm{s}$, mean gradient of $42 \mathrm{mmHg}$ and doppler velocity index of 0.25 ). Coronary angiogram showed normal coronary arteries. She was evaluated by the local heart team and TAVI was recommended due to her age and high operative risk (STS 6.2\%). Computed tomography angiogram showed a severely calcified aortic ring and ascending aorta (Figure 1), an annulus area of $418 \mathrm{~mm}^{2}$ and an average annular diameter of $23 \mathrm{~mm}$, peri-meter $71 \mathrm{~mm}$ (Figure 2). We decided for an implantation of a Portico $27 \mathrm{~mm}$ valve (Abbott Vasc, USA) via right transfemoral approach.

Transcatheter aortic valve introducer was placed through the right femoral artery and a pig-tail catheter through $6 \mathrm{~F}$ introducer in the left femoral artery. The aortic valve was initially crossed with a 0.0035 -inch straight-tip wire that was later exchanged for a 0.0035-inch Amplatz SS (Boston Scientific, Burlington, Massachusetts) using an Amplatz left (AL) 1 catheter (Launcher, Medtronic). After a pre-dilatation with a Zelos PTA $22 \times 40 \mathrm{~mm}$ balloon (OptiMed, Germany), a 27-mm self-expanding Portico valve (Abbott, Abbott Park, Illinois) was positioned across the aortic annulus and slowly released under fluoroscopic guidance. At the end of the valve opening the valve dislocated into the LVOT (Figure 3).

Low valve implantation resulted in severe paravalvular regurgitation leading to a drop in arterial pressure from $120 / 65 \mathrm{mmHg}$ to $110 / 25 \mathrm{mmHg}$. With immediate right ventricular pacing at $110 \mathrm{bpm}$ the diastolic pressure rose to 35 mmHg. In attempt to reposition the valve a $25-\mathrm{mm}$ Amplatzer Gooseneck Snare was introduced through the AL $16 \mathrm{~F}$ guide catheter (Launcher, Medtronic) 
through a contralateral femoral access and looped around the valve attachment post toward the greater curvature of the aorta. Snaring was ineffective as we obtained only a rotation of the valve frame. Therefore a second 25-mm Amplatzer Gooseneck Snare was introduced ipsilaterally through a cut AL $16 \mathrm{~F}$ guiding catheter and looped around the valve attachment post towards the smaller curvature of the aorta. Even when using two snares there was not enough support for effective pulling. We decided to add support to the second snare with an AL $16 \mathrm{~F}$ catheter. Both snares were shortened and fixed with a pean clamp (Figure $4)$.

We applied simultaneous pulling of both snares and AL 1 catheters (mother-in-child), the "double snare" technique. With exchange of tension from one snare to the other (by using steady moderate traction on one snare and progressive intermittent pulling on the other one) we were able to successfully retract and reposition the prosthesis (Figure 5).

The patient remained hemodynamically stable during the procedure and a rise in arterial pressure to $120 / 65 \mathrm{mmHg}$ was observed following valve repositioning. Targeted TTE showed normal prosthetic valve function, without paravalvular regurgitation. Aortogram demonstrated good valve position in the aortic root with no residual aortic regurgitation (Figure 6).

No tachyarrhythmias, conduction abnormalities or cerebrovascular complications were observed during the procedure and follow-up. At 1 year follow-up the patient had no complaints and denied dyspnoea or chest pain on exertion.

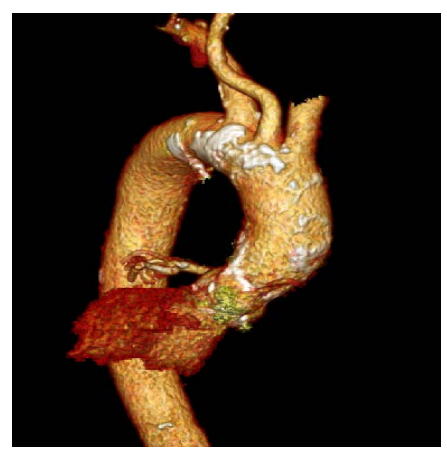

Figure 1. CT aortogram: severely calcified aortic ring and ascending aorta.
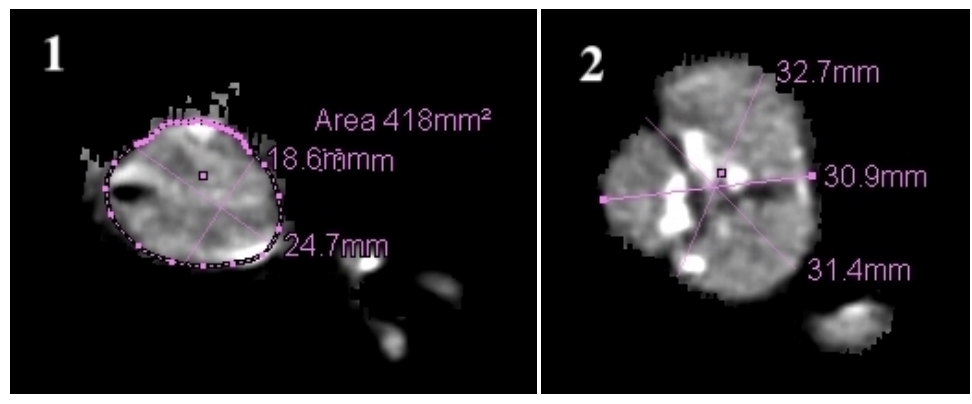

Figure 2. CT aortogram: 1) aortic annulus measurement, 2) aortic bulbus measurement. 


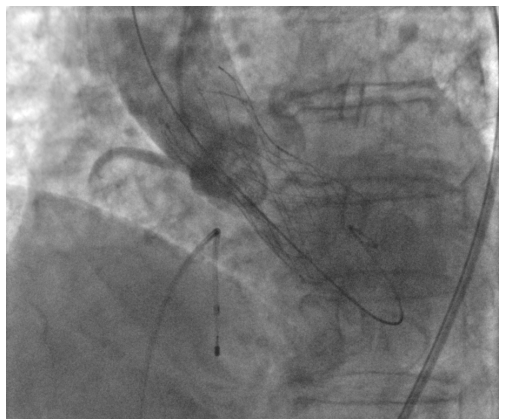

Figure 3. Portico 27-mm valve dislocation in the left ventricular outflow tract.
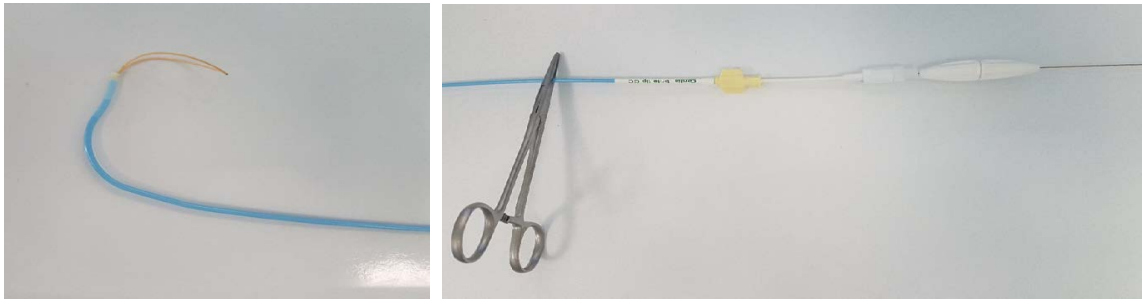

Figure 4. Snares introduced through AL 1 catheter and fixed with a pean clamp.
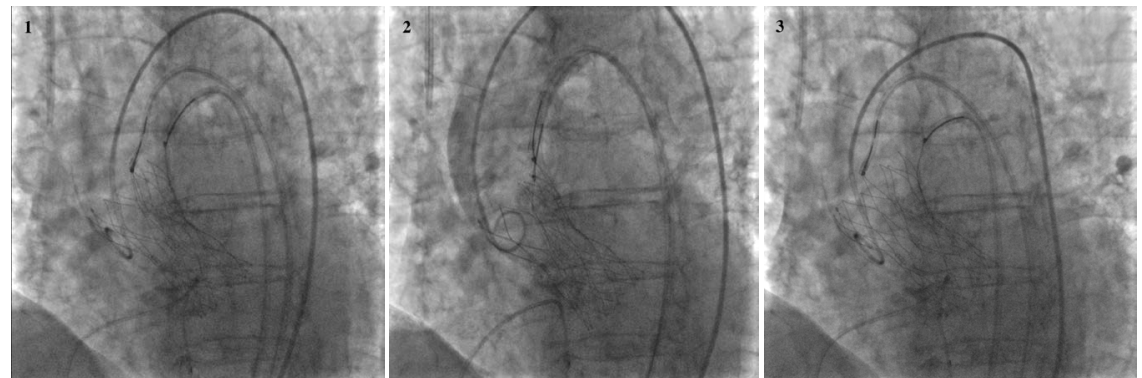

Figure 5. "Double snare" technique: two Amplatzer Gooseneck Snares were introduced through AL $16 \mathrm{~F}$ catheters (mother-in-child) (1). With exchange of tension from one snare to the other the valve was successfully repositioned (2 and 3 ).

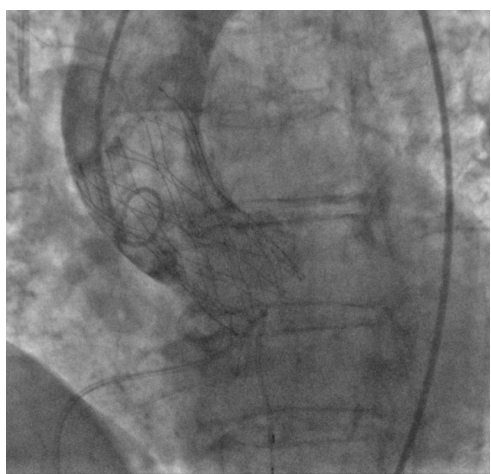

Figure 6. Final valve position with no residual regurgitation.

\section{Discussion}

Implantation of a self-expanding valve into a dense calcified aortic annulus can be challenging and may result in device malposition and malfunction. We pre- 
sented a case of deep LVOT prosthetic aortic valve dislocation with successful repositioning using a novel percutaneous "double snare" technique. Very low LVOT valve positioning leads to severe aortic regurgitation, haemodynamic instability and potential need for cardiac surgery [5]. If mild sub-annular dislocation is suspected, balloon valvuloplasty with gentle pulling of the device into the ascending aorta can be an acceptable and relatively safe repositioning strategy [4]. Recently the use of snares has been proposed via radial or transfemoral access as a bailout option for cases of severe valve dislocation. The procedure carries a high risk of failure due to difficulties in correct valve repositioning [4] [6] [7] [8]. There have been several case reports of snaring maneuvers described in the literature. Ponangi et al. reported [7] a successful use of a single snare technique for a downward dislocated Portico valve with a 25-mm Amplatzer Gooseneck Snare. However, there were no significant calcifications of the aorta present which could aggravate the repositioning procedure. Similarly, a single snare repositioning of the CoreValve system (Medtronic, Dublin, Ireland) with the use of a 35-mm Gooseneck snare was reported by Vavouranakis et al. [9]. Furthermore Beute et al. [4] managed to reposition a CoreValve system (Medtronic, Dublin, Ireland) and a newer CoreValve Evolute Pro valve (Medtronic, Dublin, Ireland) with the use of a $6 \mathrm{~F}$ EN Snare (Merit Medical System, South Jordan, UT, USA). In all previous cases the single snare technique was described as technically demanding but feasible. A technique with two snares with additional right radial access has also been proposed but not used [7]. Failure of the single snare technique in our complex case of Portico valve dislocation in the LVOT led us to use a novel, double snaring technique with two snares. The low valve dislocation was probably a result of anatomical irregularities and improper implantation steps. As severe calcifications of the aortic ring can complicate proper valve positioning the operator tried to prevent upward valve migration with gentle pushing towards the LVOT which resulted in inadvertent downward valve dislocation. Firstly, we haemodynamically stabilized the patient with ventricular pacing at $110 \mathrm{bpm}$. Attempts of repositioning the valve using the single snare technique were unsuccessful. Furthermore, the use of two snares looped around the valve attachment post was inefficient, probably due to severe aortic calcifications. The pulling strength of both snares ("double snare" technique) was improved by AL 1 catheter support (mother-in-child). Pulling force could also be improved by introducing a second snare through a right radial artery and attaching it to the smaller valve curvature attachment post. However, we decided for a different strategy and insisted on bifemoral approach. With the exchange of tension from one snare to the other we were able to retract the prosthesis towards the ascending aorta to an excellent final position at the level of the natural aortic valve.

\section{Conclusion}

It is seldom necessary to reposition a self-expanding transcatheter aortic valve 
following deployment. However, when needed, few options are available to snare the valve in order to achieve proper positioning. To our knowledge, we are the first to describe a transcatheter aortic valve repositioning using two snares. The "double snare" technique may be used as an effective repositioning strategy for deeply implanted transcatheter self-expanding aortic valves, especially in cases of severe aortic ring calcifications and ineffective single snare techniques.

\section{Disclosures}

There are no relationships with industry related to this article.

\section{Informed Consent}

The patient discussed in the following case has given an informed consent.

\section{Sources of Funding}

None.

\section{Conflicts of Interest}

The authors declare no conflicts of interest regarding the publication of this paper.

\section{References}

[1] Ussia, G.P., Barbanti, M, Immè, S., et al. (2010) Management of Implant Failure during Transcatheter Aortic Valve Implantation. Catheterization and Cardiovascular Intervention, 76, 440-449. https://doi.org/10.1002/ccd.22595

[2] Eggebrecht, H., Schmermund, A., Kahlert, P., et al. (2013) Emergent Cardiac Surgery during Transcatheter Aortic Valve Implantation (TAVI): A Weighted Meta-Analysis of 9,251 Patients from 46 Studies. EuroIntervention, 8, 1072-1080. https://doi.org/10.4244/EIJV8I9A164

[3] Gorla, R., De Marco, F., Morganti, S., et al. (2020) Transcatheter Aortic Valve Implantation with the Portico and Evolut R bioprostheses in Patients with Elliptic Aortic Annulus. Eurointervention: Journal of Europcr in Collaboration with the Working Group on Interventional Cardiology of the European Society of Cardiology, 15, e1588-e1591. https://doi.org/10.4244/EIJ-D-19-00115

[4] Beute, T.J., Nolan, M.A., Merhi, W.M. and Leung Wai Sang, S. (2018) Use of EN Snare Device for Successful Repositioning of the Newest Self-Expanding Transcatheter Heart Valve. SAGE Open Medical Case Reports. https://doi.org/10.1177/2050313X18819933

[5] Geisbüsch, S., Bleiziffer, S., Mazzitelli, D., et al. (2010) Incidence and Management of CoreValve Dislocation during Transcatheter Aortic Valve Implantation. Circulation: Cardiovascular Interventions, 3, 531-536. https://doi.org/10.1161/CIRCINTERVENTIONS.110.944983

[6] Valkov, V., Kalchev, D., Kostadinov, A., et al. (2018) Snare Maneuver for Management of Low TAVI Implantation-Clinical Case. Journal of IMAB-Annual Proceeding Scientific Papers, 24, 2034-2037. https://doi.org/10.5272/jimab.2018242.2034

[7] Ponangi, U.P., Yadav, S., Kushwaha, V., et al. (2019) Correction of a Malpositioned 
Portico Transcatheter Aortic Valve Using a Snare Maneuver. JACC: Case Reports, 1, 838-843. https://doi.org/10.1016/j.jaccas.2019.11.023

[8] Yildiz, B.S., Alihanoglu, Y.I., Alur, I., et al. (2015) A Rare Complication: An Attempt of Retrieval of an Aortic Valve Wrapped with Pig Tail Catheter during Transcatheter Aortic Valve Implantation. Cardiovascular Revascularization Medicine, 16, 376-377. https://doi.org/10.1016/j.carrev.2015.06.004

[9] Vavouranakis, M., Vrachatis, D.A., Toutouzas, K.P., et al. (2010) “Bail Out” Procedures for Malpositioning of Aortic Valve Prosthesis (Core Valve). International Journal of Cardiology, 145, 154-155. https://doi.org/10.1016/j.ijcard.2009.07.040 